\title{
Evaluation of Encephaloduroarteriosynangiosis Efficacy Using Probabilistic Independent Component Analysis Applied to Dynamic Susceptibility Contrast Perfusion MRI
}

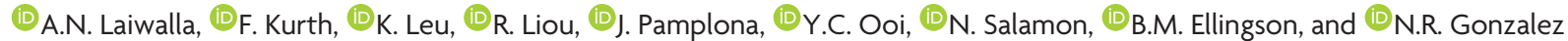

\begin{abstract}
BACKGROUND AND PURPOSE: Indirect cerebral revascularization has been successfully used for treatment in Moyamoya disease and symptomatic intracranial atherosclerosis. While angiographic neovascularization has been demonstrated after surgery, measurements of local tissue perfusion are scarce and may not reflect the reported successful clinical outcomes. We investigated probabilistic independent component analysis and conventional perfusion parameters from DSC-MR imaging to measure postsurgical changes in tissue perfusion.

MATERIALS AND METHODS: In this prospective study, 13 patients underwent unilateral indirect cerebral revascularization and DSC-MR imaging before and after surgery. Conventional perfusion parameters (relative cerebral blood volume, relative cerebral blood flow, and TTP) and probabilistic independent components that reflect the relative contributions of DSC signals consistent with arterial, capillary, and venous hemodynamics were calculated and examined for significant changes after surgery. Results were compared with postsurgical DSA studies to determine whether changes in tissue perfusion were due to postsurgical neovascularization.

RESULTS: Before surgery, tissue within the affected hemisphere demonstrated a high probability for hemodynamics consistent with venous flow and a low probability for hemodynamics consistent with arterial flow, whereas the contralateral control hemisphere demonstrated the reverse. Consistent with symptomatic improvement, the probability for venous hemodynamics within the affected hemisphere decreased with time after surgery $(P=.002)$. No other perfusion parameters demonstrated this association. Postsurgical DSA revealed an association between an increased preoperative venous probability in the symptomatic hemisphere and neovascularization after surgery.
\end{abstract}

CONCLUSIONS: Probabilistic independent component analysis yielded sensitive measurements of changes in local tissue perfusion that may be associated with newly formed vasculature after indirect cerebral revascularization surgery.

ABBREVIATIONS: EDAS = encephaloduroarteriosynangiosis; ICA = independent component analysis; ICAS = intracranial atherosclerosis; $\mathrm{MMD}=$ Moyamoya disease; $\mathrm{rCBF}=$ relative cerebral blood flow; $\mathrm{rCBV}=$ relative cerebral blood volume

$E^{\mathrm{n}}$ ncephaloduroarteriosynangiosis (EDAS) is a form of indirect cerebral revascularization, which has been successfully used as a treatment in Moyamoya disease (MMD) and symptomatic intracranial atherosclerosis (ICAS) in adults. ${ }^{1-8}$ In principle, extracranial arterial branches are surgically redirected below the

Received November 17, 2015; accepted after revision October 17, 2016.

From the Departments of Neurosurgery (A.N.L., Y.C.O.) and Radiology (K.L., J.P., N.S., B.M.E.), David Geffen School of Medicine, University of California, Los Angeles, Los Angeles, California; and Department of Neurosurgery (F.K., R.L., N.R.G.), Cedars

Sinai Medical Center, Los Angeles, California.

This work is supported by the Ruth and Raymond Stotter Endowed Chair in Neurosurgery and the National Institutes of Health award K23NS079477.

Please address correspondence to Nestor R. Gonzalez, MD Cedars Sinai Medical Center, 127 S San Vicente Blvd, Suite A6600, Los Angeles, CA 90048 ,

e-mail: nestor.gonzalez@cshs.org

-- Indicates open access to non-subscribers at www.ajnr.org

7 Indicates article with supplemental on-line photos.

http://dx.doi.org/10.3174/ajnr.A5041 dura mater and apposed to hypoperfused brain tissue. This procedure results in the sprouting of new vessels that gradually form new anastomoses with the intracranial circulation, improving perfusion and reducing the risk of stroke. ${ }^{7}$ In a recently published report on treatment outcomes with EDAS, the rate of stroke was $2.4 \%$ (5.6\% in patients with ICAS, $0 \%$ in patients with MMD) within 2 years after the operation. ${ }^{9}$ These rates are lower than the previously observed rates for medical treatment (15\%) or angioplasty and stent placement (21\%) in the SAMMPRIS (Stenting vs. Aggressive Medical Management for Preventing Recurrent Stroke in Intracranial Stenosis) trial. ${ }^{10,11}$

Multiple imaging modalities have been used to assess the extent of indirect revascularization in patients with MMD and ICAS. Conventional angiography, CTA, and MRA have demonstrated neovascularization within the EDAS operative territory through transdural and transpial collateral vessels. ${ }^{12,13}$ Increased donor superficial temporal artery and middle meningeal artery vessel sizes and a decrease in preoperative native collaterals fol- 


\begin{tabular}{|c|c|c|c|c|c|c|c|}
\hline \multirow[b]{2}{*}{ ID } & \multirow[b]{2}{*}{$\begin{array}{l}\text { Age } \\
\text { (yr) }\end{array}$} & \multirow[b]{2}{*}{ Sex } & \multirow[b]{2}{*}{ Diagnosis } & \multicolumn{2}{|c|}{$\begin{array}{l}\text { Events Pre- and } \\
\text { Postoperation }\end{array}$} & \multicolumn{2}{|c|}{ Imaging Data } \\
\hline & & & & Pre- & Post- & $\begin{array}{l}\text { Time Range } \\
\text { (days) }\end{array}$ & $\begin{array}{l}\text { Time } \\
\text { Points }\end{array}$ \\
\hline 1 & 55 & $\mathrm{~F}$ & ICAS & TIA & & $-19-373$ & 5 \\
\hline 2 & 65 & $\mathrm{~F}$ & ICAS & Stroke & TIA & $-14-364$ & 4 \\
\hline 3 & 75 & $\mathrm{~F}$ & ICAS & Stroke & & $107-365$ & 4 \\
\hline 4 & 66 & $\mathrm{~F}$ & ICAS & TIA + Stroke & & 99-372 & 4 \\
\hline 5 & 63 & $M$ & ICAS & Stroke & & $-14-370$ & 4 \\
\hline 6 & 55 & $\mathrm{~F}$ & MMD & Stroke & & $-71-172$ & 3 \\
\hline 7 & 23 & $\mathrm{~F}$ & MMD & Stroke & & $-4-94$ & 2 \\
\hline 8 & 67 & $M$ & ICAS & TIA & & $-7-190$ & 2 \\
\hline 9 & 56 & $\mathrm{~F}$ & MMD & TIA & & $-7-135$ & 2 \\
\hline 10 & 47 & $\mathrm{~F}$ & ICAS & Stroke & & $-3-186$ & 3 \\
\hline 11 & 70 & $\mathrm{~F}$ & ICAS & TIA + Stroke & & -15 & 1 \\
\hline 12 & 35 & $\mathrm{~F}$ & MMD & TIA + Stroke & & 116 & 1 \\
\hline 13 & 35 & $\mathrm{~F}$ & MMD & TIA & & 437 & 1 \\
\hline
\end{tabular}

Note:-ID indicates identification.

lowing EDAS have also been reported..$^{5,12,14,15}$ Beyond anatomic changes, investigating tissue perfusion is necessary because alterations in tissue perfusion are dynamic processes that may not correlate directly with structural changes. ${ }^{16,17}$ In MMD, xenonenhanced CT scanning, SPECT, blood oxygen level-dependent cerebrovascular-reactivity MR imaging, and PET studies have shown improved cerebrovascular reactivity in territories of the intervened hemisphere. ${ }^{12,18,19}$ In addition, CT perfusion and DSC-MR imaging studies found improved postoperative cerebral hemodynamics through TTP, relative cerebral blood flow (rCBF), and relative cerebral blood volume (rCBV) measures. ${ }^{16,20}$ Yet, the association between local tissue perfusion following EDAS with structural changes and clinical outcomes is still unclear.

Probabilistic independent component analysis (ICA) of DSC-MR imaging allows improved differentiation of physiologic and pathophysiologic cerebral hemodynamics through the extraction of spatiotemporal blood supply patterns. ${ }^{21}$ It is a datadriven, multivariate approach that has been applied to functional MR imaging, electroencephalographic recordings, and, recently, DSC-MR imaging data in glioblastoma, in which it demonstrated the ability to discern tumor from normal vasculature and characterize tumor perfusion patterns. ${ }^{22-27}$ The objective of this study was to apply ICA to DSC-MR imaging data to evaluate hemodynamic changes after EDAS, to assess whether this method can detect changes consistent with structural imaging measures, and to compare the results with classic perfusion measures - that is, by quantitatively separating the DSC-MR imaging time-series data in each image voxel into independent temporal patterns consistent with arterial, capillary, and venous hemodynamics, we hypothesized that probabilistic ICA would detect subtle changes in vascularity that may not be captured by classic perfusion measures. We further hypothesized that brain regions in adults with symptomatic ICAS or MMD would contain tissue with a high venous probability, suggesting hemodynamics consistent with delayed venous flow. Additionally, we posited that a favorable increase in tissue perfusion within the surgical hemisphere after EDAS would reduce this venous probability and that this reduc- tion would be associated with neovascularization as measured with postoperative digital subtraction angiography.

\section{MATERIALS AND METHODS Study Design}

We performed a prospective study of adult patients (23-75 years of age) with TIA or nonsevere stroke attributed to $70 \%-99 \%$ intracranial stenosis of a major intracranial artery and confirmed by conventional angiography. All patients presented with persistent strokes or TIAs despite optimal medical management, as established in the SAMMPRIS trial. ${ }^{10} \mathrm{~Pa}$ tients with ICAS or MMD were included in this study, with the former enrolled in the ongoing EDAS Surgical Indirect Revascularization for Symptomatic Intracranial Arterial Stenosis (ERSIAS) trial (clinicaltrials.gov No. NCT01819597). Patients with MMD received the same imaging and therapy as those with ICAS through the ERSIAS protocol. All participants provided informed consent before taking part, and the study was conducted with institutional review board approval (No. 12-000439; University of California, Los Angeles).

\section{Study Participants}

Thirteen patients (female/male ratio: 11:2; mean age, $54.77 \pm 15.66$ years) who underwent unilateral EDAS surgery and DSC-MR imaging from 2013 to 2014 were included (see Table 1 for an overview of all enrolled patients). Eight of the patients were diagnosed with ICAS, and 5, with MMD. Of the 13 patients, 8 had DSC-MR imaging data at multiple time points, including 1 pre- and up to 4 postoperatively (ie, at approximately 3, 6, 9, and 12 months after the operation). The exact timing of the imaging studies was recorded in days after the operation, yielding negative numbers for preoperative scans (Table 1). For 12 of the 13 patients, postoperative DSA studies were performed to document the extent of newly formed vessels from the donor artery. These studies were used to compare potential perfusion changes with the actual observed sprouting of new vessels. One patient (patient 11) died of a myocardial infarction before these postoperative studies were performed.

\section{Preoperative Evaluation}

All patients underwent preoperative imaging that included DSA and/or MR imaging. DSA of all patients revealed severe stenosis in the intracranial cerebral vasculature that corresponded to their clinical symptoms. EDAS was considered for treatment on the basis of clinical need, including ischemic symptoms within 30 days and evidence of hypoperfusion and poor collateral flow (American Society of Interventional and Therapeutic Neuroradiology/Society of Interventional Radiology grades $0-2$ ).

\section{Surgical Technique}

The EDAS surgical technique has been previously described. ${ }^{5,7}$ In brief, the superficial temporal artery is identified and dissected with its surrounding galeal cuff. A craniotomy is performed, and the dura 
is opened in cruciate fashion, preserving the middle meningeal artery branches. The dural layers are separated, the inner avascular layer is removed, and the arachnoid is widely opened with microdissection under the surgical microscope. The superficial temporal artery is subsequently sutured to the edges. The burr-holes and inner table of the bone flap are trimmed to prevent kinking and compression of the superficial temporal artery on its replacement. Patients remain in intensive medical management during and after the operation with antiplatelets, high-dose statins, and strict control of blood pressure and glycemia.

\section{MR Images}

All MR imaging data were collected on a 1.5T (Avanto; Siemens, Erlangen, Germany) or 3T MR imaging system (Trio, Prisma, or Skyra; Siemens). Each patient underwent follow-up studies on the same MR imaging scanner that was used for their original preoperative baseline scan. Standard anatomic MR imaging sequences included a sagittal T1-weighted image, along with axial T2-weighted and FLAIR images. A 10- to $20-\mathrm{mL}(0.1 \mathrm{mmol} / \mathrm{kg}$ body weight $)$ dose of gadobenate dimeglumine contrast agent was administered during DSC-MR imaging (gradient-echo EPI: TE/TR $=23 / 1210 \mathrm{~ms}$ [1.5T], TE/ $\mathrm{TR}=32 / 1840 \mathrm{~ms}[3 \mathrm{~T}], 35^{\circ}$ flip angle, 120 time points, bolus injection after 20-25 baseline images, 16-20 sections, 5-mm section thickness with no intersection gap, $128 \times 128$ matrix size, 24-cm FOV). rCBV, rCBF, and TTP were computed via the commercially available postprocessing software (IB Neuro, Version 2.0; Imaging Biometrics, Elm Grove, Wisconsin). Sagittal and axial postcontrast T1-weighted images were obtained following DSC perfusion images.

\section{Probabilistic Independent Component Analysis}

Technical and mathematic details regarding the probabilistic ICA algorithm are described in Beckmann and Smith. ${ }^{28}$ In the current study, we implemented the probabilistic ICA algorithm by using the MELODIC program from the FMRIB Software Library (FSL; http://fsl.fmrib.ox.ac.uk/fsl/fslwiki/ MELODIC). Briefly, probabilistic ICA was applied to the raw DSC-MR imaging data throughout the entire brain to identify 3 statistically independent temporal components plus noise. ${ }^{22}$ These 3 independent components were then manually identified as "arterial," "venous," and "capillary" components based on their temporal hemodynamic profiles, in which the arterial component had the shortest TTP, the venous component had the longest TTP, and the capillary component had the smallest signal amplitude and an intermediate TTP. For verification of this empiric categorization, the components were checked via their respective spatial patterns to ensure that the arterial component covered the circle of Willis and the venous component contained the choroidal plexus. Next, the posterior probability of the DSC time signal for each independent component compared with noise was calculated for each image voxel. This calculation resulted in 3 unique brain maps reflecting the relative probability of a voxel having a DSC time-series consistent with arterial, venous, or capillary hemodynamic profiles. These resulting probability maps were then used for subsequent analyses.

\section{Image Registration}

All conventional and perfusion images for each subject were registered to the axial, postcontrast T1-weighted images at the first postoperative time point by using a $12-d f$ affine transformation with a mutual information cost function (in FSL). If required, 9- $d f$ manual alignment was subsequently performed (tkregister2, FreeSurfer; surfer.nmr.mgh.harvard.edu). Three investigators (A.N.L., B.M.E., K.L.) verified adequate alignment of the images by consensus.

\section{ROls}

ROIs were manually drawn on baseline postcontrast T1-weighted images around the surgical graft site and on a mirrored region within the contralateral hemisphere. Because all images were aligned between baseline and postsurgical follow-up, a single set of ROIs was used for comparison. The mean values of all 6 measures (arterial probability, capillary probability, venous probability, rCBV, rCBF, and TTP) within these 2 ROIs (surgical and contralateral) were calculated for each patient and evaluation time point.

\section{Statistical Analysis}

The calculated ROI-wise values (probabilities for arterial, capillary, and venous components, as well as $\mathrm{rCBV}, \mathrm{rCBF}$, and TTP) were used as the dependent measures in a statistical model. The association between time after the operation (independent variable) and the imaging measures of interest (dependent variable) was calculated by using a general linear model and permutation testing. This approach allowed accounting for interindividual differences among the participants by including "subject" as a factor of no interest and assessing interactions between surgical and control hemispheres by modeling appropriate interactions. Correlation coefficients were calculated from the $t$ values on the basis of the effective $d f s$. Because none of the measures of interest can be assumed to be normally distributed in this small sample, significance was established by using a Monte Carlo simulation with 10,000 permutations. ${ }^{29}$ Specifically, the null hypothesis was that there is no association between the time after the operation and the measures of interest (dependent variables), so all observed differences were explained by interindividual variability and noise. This entails that no systematic changes occur in either measurement with time, which means that under the null hypothesis it is inconsequential at which time relative to surgery the measure was taken. While we controlled for interindividual differences, the time after the operation can thus be interchanged at random, without yielding a less significant correlation or interaction under the null hypothesis. The null hypothesis can be rejected at $P \leq .05$, however, if random permutations of the time after the operation yield less significant results in at least $95 \%$ of all cases. With 6 different measures of interest, we controlled the type I error by applying a Bonferroni correction for multiple comparisons (ie, significance was determined at $P<.0083$ ). Seeing that previous analyses focused on MMD, and not ICAS, and that the underlying disease may be a modifying factor for the results, we repeated the statistical analysis for each disease group as a post hoc analysis and additionally calculated the interaction between disease group and 


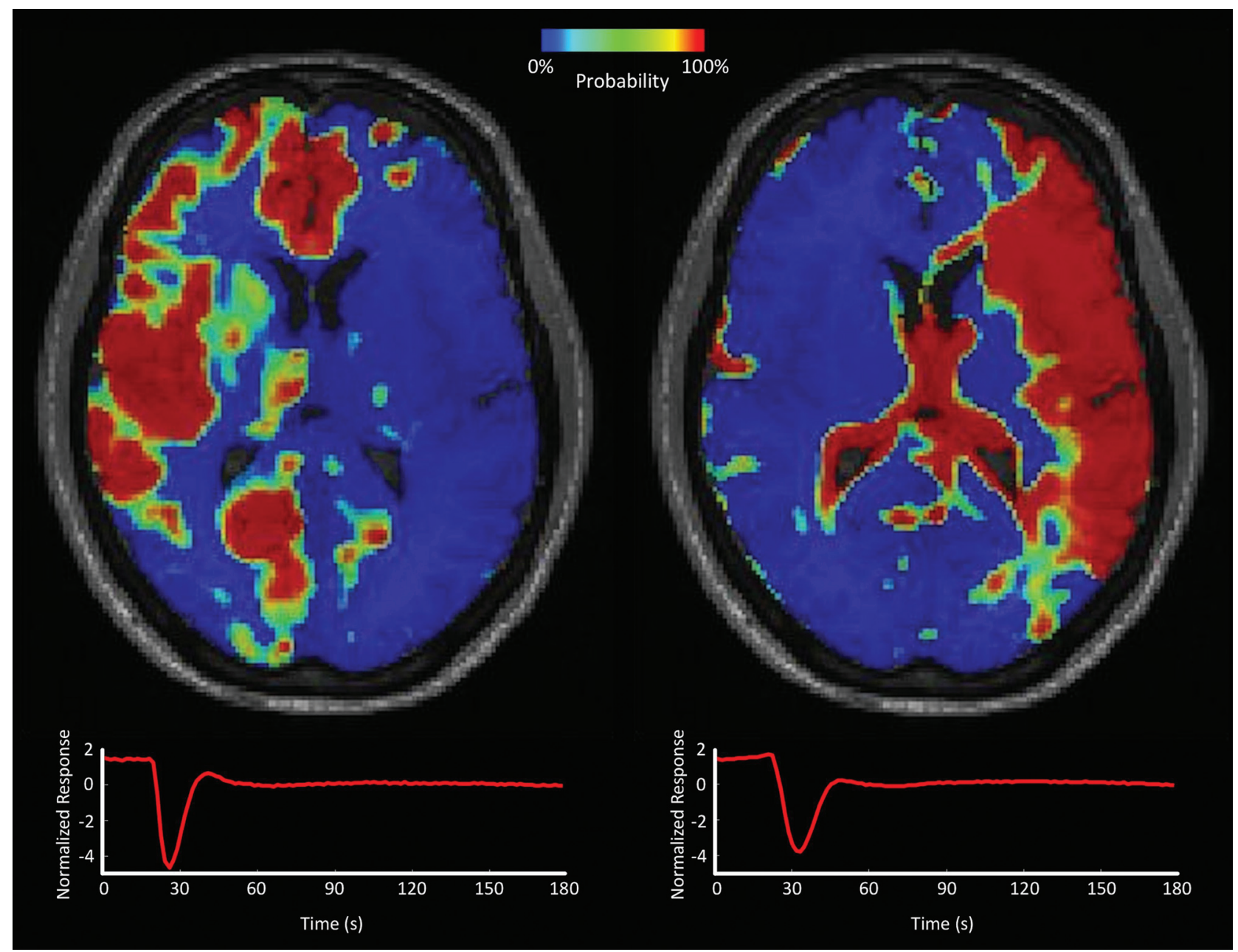

FIG 1. Probabilistic independent component analysis of presurgical DSC-MR imaging of a patient undergoing EDAS. Probability maps of arterial (left) and venous (right) components are shown on an axial section. The respective time courses show a delayed response in the venous component. The color bar indicates local probability. Note the high probability for the venous component in the left hemispheric cortex, which is the symptomatic side.

postsurgical changes. The analysis was performed in Matlab (Version 7.12; MathWorks, Natick, Massachusetts).

In addition, standard plots of the probability of arterial, venous, and capillary components within the prescribed ROIs with time in the complete sample and descriptive statistics were generated for demographic and clinical assessments to characterize the study population. Additional plots were created for $\mathrm{rCBF}, \mathrm{rCBV}$, and TTP. For comparison with the individual DSA studies, plots of the changes within the arterial and venous component with time for each individual patient were created. These analyses were conducted by using JMP (Version 11; SAS Institute, Cary, North Carolina).

\section{Clinical Analysis}

Patient data were reviewed up to 24 months following EDAS surgery to evaluate TIA, stroke, and death.

\section{Role of the Funding Source}

None of the funding sources had any role in the collection or interpretation of data or any influence on the writing or decision to submit the manuscript.

\section{RESULTS}

Probabilistic ICA of the asymptomatic control hemisphere revealed a lower probability of belonging to the venous component than to the arterial component at baseline (mean probability: arterial, $0.40 \pm 0.08$, versus venous, $0.16 \pm 0.07$ ). In the preoperative symptomatic hemisphere, the probabilities of arterial and venous components were inverted (mean probability: venous, $0.46 \pm 0.19$, versus arterial, $0.23 \pm 0.09$ ). This inversion is depicted in the preoperative probabilistic map of arterial and venous components shown in Fig 1 for a representative patient. Visually, the maps showed striking asymmetry between the right and left frontal cortices, with a higher venous probability in the left frontoparietal cortex (symptomatic site) compared with contralateral tissue. Images for several axial sections of this patient are shown in On-line Fig 1.

With time, baseline values of venous and arterial probabilities were maintained in the asymptomatic control side (Fig 2). There was no significant correlation between the time after the operation and the venous probability of this asymptomatic hemisphere. After the operation, however, the high venous probabilities of the operative side (preoperative mean probability, $0.46 \pm 0.19$, versus 

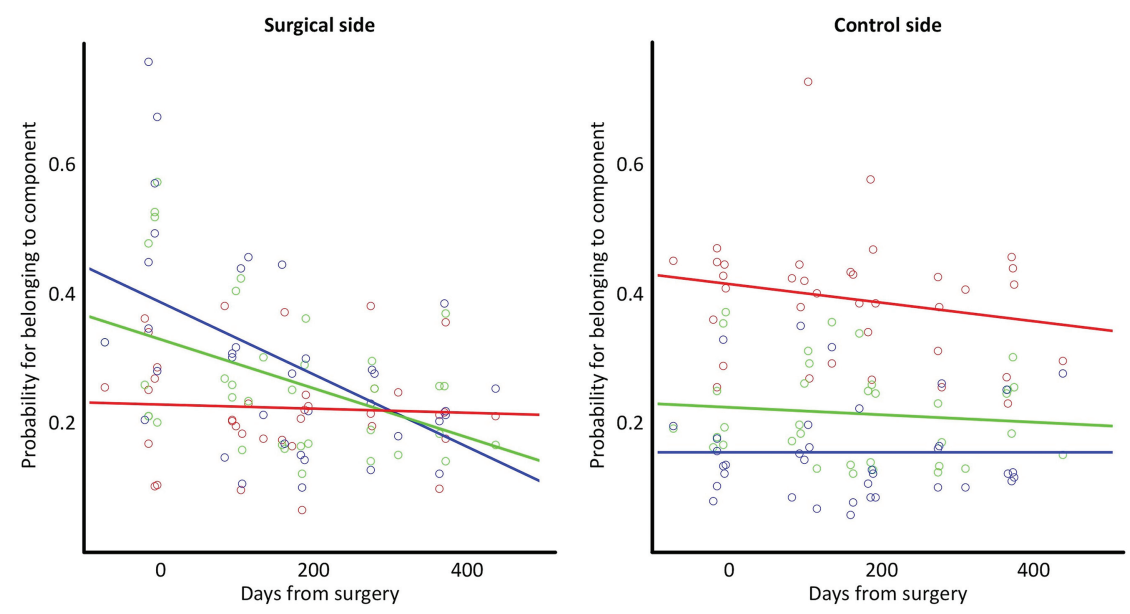

subgroup and time after the operation did not yield any significant results, thus not supporting a difference between ICAS and MMD. Notably, the study was not designed to separately evaluate each etiology group; however, the striking magnitude of the effect of the operation in the ICAS group reached statistical significance in this post hoc analysis. No other perfusion measure showed significant changes after the operation in that subgroup analysis.

Exploration of individual arterial and venous components in the surgical hemispheres of each patient did not demonstrate the pattern of increased venous probability across all patients. Of all 13 patients, 3 did not show an increased venous probability preoperatively. Fig 3 depicts examples from 1 patient with and 1 patient without increased venous proba-

FiG 2. Temporal changes in the mean local probability of the tissue to belong to either arterial (red), venous (blue), or capillary (green) component. The dominance of the venous component at baseline in the symptomatic hemisphere indicates delayed perfusion. Note the normalization in dominance with time, suggesting an accelerated perfusion of the surgical region.

\begin{tabular}{|c|c|c|c|c|c|}
\hline & $\begin{array}{c}P \\
\text { Interaction }\end{array}$ & $\begin{array}{c}r \\
\text { Surgical } \\
\text { Side }\end{array}$ & $\begin{array}{c}P \\
\text { Surgical } \\
\text { Side }\end{array}$ & $\begin{array}{c}r \\
\text { Control } \\
\text { Side }\end{array}$ & $\begin{array}{c}P \\
\text { Control } \\
\text { Side }\end{array}$ \\
\hline Artery & .258 & 0.024 & .418 & -0.109 & .194 \\
\hline Vein & $.001^{a}$ & -0.426 & $.002^{\mathrm{a}}$ & 0.051 & .281 \\
\hline Capillary & .009 & -0.332 & .020 & -0.003 & .489 \\
\hline $\mathrm{rCBF}$ & .270 & -0.017 & .444 & -0.116 & .226 \\
\hline $\mathrm{rCBV}$ & .404 & 0.157 & .121 & 0.113 & .192 \\
\hline TTP & .120 & 0.181 & .151 & 0.260 & .039 \\
\hline
\end{tabular}

Note:- $P$ indicates probability; $r$, correlation coefficient.

${ }^{\text {a }}$ Significant results after correction for multiple comparisons using a Bonferroni correction

12-month probability, $0.23 \pm 0.09$ ) decreased with time, while the arterial probabilities remained largely unchanged. This outcome was also reflected in a significant negative correlation between the time after the operation and the venous probability of this symptomatic hemisphere $(P=.002)$. The association between venous probability and the time after the operation was significantly stronger on the surgical side compared with the control side $(P=.001)$, in which no significant correlation between any perfusion measure and time after the operation was detected. After approximately 9 months, probabilities of the individual components of the surgical side appeared inverted to reflect patterns similar to those of the nonsurgical side. No other interactions between hemispheres (control versus surgical) or correlations with time after the operation survived the correction for multiple comparisons for any other perfusion measurement. Table 2 provides a detailed overview of $P$ values and correlation coefficients. Although no significant findings were observed for the classic measures, rCBV, rCBF, and TTP, the respective plots can be found in On-line Fig 2 for completeness.

Post hoc analyses for each etiology group revealed a significant negative correlation between the venous probability and time after the operation of the surgical hemisphere in patients with ICAS $(P=.004)$; this correlation was not significant for patients with MMD, likely due to the small sample size. An interaction between bility. A direct comparison with postoperative DSA studies revealed that the 3 patients who did not show increased venous probabilities preoperatively did not develop new collaterals from the EDAS surgery. However, all other patients who had an inversion of the components did develop new collaterals from the donor vessel after the operation. This observation suggests that measures of venous probability may be a surrogate for neovascularization. An overview of the direct comparisons in all patients is given in On-line Fig 3.

\section{Clinical Results}

No patient had a stroke within the 24-month follow-up period. The 3 patients who did not develop postoperative collaterals had improvement in the degree of intracranial arterial stenosis and did not develop strokes; this outcome was attributed to the medical management. One of the 14 patients (patient 2) had a TIA at 1-6 months postoperatively. Further TIAs were not observed during the follow-up period for this patient. None of the other 12 patients had a TIA postoperatively.

\section{DISCUSSION}

Collaterals are of critical importance in maintaining cerebral perfusion in patients with intracranial arterial stenosis. ${ }^{30}$ EDAS facilitates the formation of new collaterals between redirected extracranial arterial branches and the intracranial circulation. ${ }^{7}$ The extent of revascularization following EDAS is traditionally focused on the anatomic investigation of collaterals by conventional angiography. However, consideration of the functional effects is also necessary because alterations in tissue perfusion may not correlate directly with structural changes. ${ }^{16,17,31,32}$ The goal of this study was to explore perfusion MR imaging measurements to better characterize tissue perfusion following EDAS. No significant relationships were found between conventional perfusion measures (rCBV, rCBF, and TTP) and the time after EDAS surgery. By applying probabilistic ICA, however, a significant change was observed in the surgical hemisphere that corresponded to the clinical observation of protection from stroke and a reduced risk 

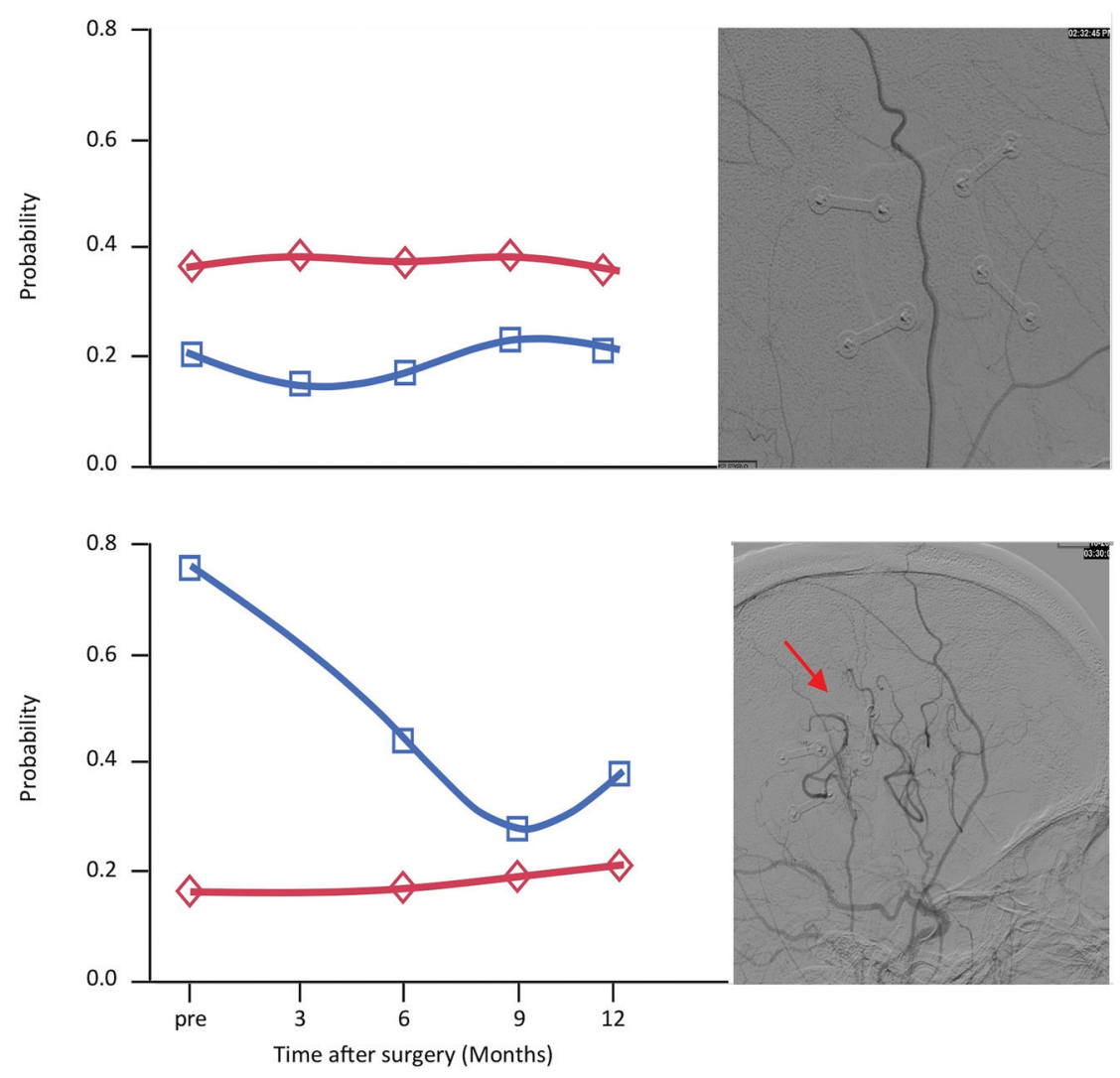

FIG 3. Comparison between the results from probabilistic ICA and the postoperative DSA studies in 2 sample patients. The results for the arterial and venous component are shown on the left, with the arterial component in red and the venous component in blue. The sagittal view of the respective postoperative DSA study is depicted directly to the right side of these graphs. Upper part: Note that this patient did not exhibit the pattern of an inversion of the probabilities as presented in Fig 2 for the whole sample. The respective postoperative DSA study does not show any new anastomoses between the donor artery and the intracranial vasculature. Lower part: This patient exhibited an inversion of the probabilities on the surgical side with a postoperative decrease of the venous component. The newly formed anastomoses with the intracranial arteries are indicated with a red arrow.

of TIA after EDAS, in agreement with prior publications reporting these beneficial clinical effects. ${ }^{7,9}$ Specifically, the nonsurgical (control) hemisphere was found to have a constant, high arterial probability and low venous and capillary probabilities, suggesting that the raw DSC signal was most consistent with a rapid arterial hemodynamic pattern. Because this asymptomatic hemisphere was not affected by intracranial arterial stenosis, no perfusion deficit was expected.

In contrast, the symptomatic hemisphere, in which there was intracranial arterial stenosis and thus made the patient eligible for EDAS revascularization, had high venous and capillary probabilities and a low arterial probability at baseline, suggesting that most tissue near the surgical site expressed patterns more similar to delayed venous hemodynamics. This shift toward a higher probability of the venous component within the symptomatic hemisphere can be interpreted as a slower perfusion in this region. After EDAS surgery, the elevated probability for the venous component significantly decreased with time; this decrease led to an inversion of the probability pattern at 9 months. This suggests a normalization of the blood flow characteristics, with faster arrival and clearing of the contrast agent in the tissue due to higher perfusion after EDAS. In other words, after EDAS surgery, tissue perfusion changes in that it becomes less delayed than before the operation, thus less resembling venous blood flow characteristics. This interpretation is consistent with the clinical observation that symptoms improved in all cases following EDAS surgery.

While we also observed a significant negative correlation between the time after the operation and venous probability in the surgical hemisphere within the ICAS group, no significant correlation between the time after the operation and any perfusion measure was found within the MMD group. The interaction between patient group and time after the operation was not significant, indicating that the response to the operation did not differ between patients with ICAS and those with MMD. Nonsignificant results within the MMD group may rather be due to the relatively small sample size; this study was not designed to investigate each group independently. As shown in Table 1 , the MMD group comprised only 5 of the 13 patients, and future analysis based on a larger dataset is desirable to further evaluate these findings.

The comparison with postoperative DSA studies confirms that improvement in perfusion was associated with formation of new collaterals. Patients who did not develop new collaterals from the donor vessel did not show an increased venous probability in the surgical hemisphere according to the results from the probabilistic ICA. These patients also exhibited a reduction in the stenosis of the qualifying artery in DSA angiography with time, which may be why they did not develop new collaterals, because the innate source of cerebral irrigation was sufficient to prevent them from developing symptoms. This effect has been reported in cases of intensive medical management alone, which was maintained in all patients undergoing EDAS in this study. ${ }^{11,33}$ While this observation supports the notion that the decrease in the venous probability derived from probabilistic ICA acts as a surrogate for revascularization, it raises a second interesting question: One might inquire whether the observed relationship between increased venous probability and postoperative revascularization might qualify as a marker for patient selection and/or serve as a valuable tool for surgical planning and monitoring. While this question is beyond the scope of this initial study, it is well worth following up in a larger systematic study. Furthermore, because the clinical outcome after EDAS is likely to be superior to medical therapy alone, it will be important to assess whether such an observation may be reason enough for withholding or delaying an operation. ${ }^{9-11}$

In the setting of MMD, improved cerebral perfusion following indirect revascularization has been demonstrated by using tradi- 
tional neuroimaging modalities. In a recent study with 12 pediatric patients with MMD, SPECT revealed improvement in basal perfusion and cerebrovascular reactivity in the MCA surgical territory, approximately 3 months following revascularization by EDAS surgery. ${ }^{34}$ In a similar study in 17 pediatric patients with MMD who underwent EDAS, SPECT at approximately 5 months (153 \pm 110 days) showed significant improvement in the cerebrovascular reserve, with nonsignificant improvement of the basal CBF. ${ }^{19}$ Furthermore, perfusion MR imaging in 13 children with MMD demonstrated delayed presurgical TTP enhancement compared with controls, which was significantly reduced following EDAS. ${ }^{16} \mathrm{CT}$ perfusion was also used to evaluate multiple burrhole revascularization in ischemic adult MMD. ${ }^{20}$ Six-month follow-up CT perfusion showed postoperative increases in CBF, with decreases in MTT and TTP. Our results, however, do not demonstrate the same magnitude of change in traditional perfusion measures in patients undergoing EDAS. Some of the differences can be expected because most data in this study stem from adult patients with ICAS, usually of advanced age, in whom extensive neovascularization after EDAS is not necessarily anticipated. In addition, the relatively small overall sample size and limited coverage of time points may have contributed to the nonsignificant findings with conventional perfusion measures. The significant results found with probabilistic ICA may indicate a higher sensitivity of this technique to perfusion changes, warranting further studies to validate its use to monitor EDAS effects and potentially as a selection tool to identify subjects who would benefit most from indirect revascularization.

First developed in the engineering field of signal processing, ICA has been applied to fMRI for the separation of functional networks in task-related and task-free fMRI studies and to EEG to resolve differences between evoked responses. ${ }^{25,27,35-38}$ More recently, ICA has been used to separate and distinguish phases of cerebral perfusion. Separation of the mixed dynamic signals of DSC-MR imaging into independent source signals provides spatiotemporal hemodynamic information on local perfusion for cerebral vasculature and parenchyma. ${ }^{37,39}$ In a study of 12 patients with unilateral carotid stenosis, this method differentiated normal arterial, stenotic arterial, and stenotic-side parenchymal phases; this finding suggests that stenosis and poor collateral circulation resulted in delayed contrast perfusion through parenchyma on the side of the stenosis. ${ }^{37}$ Additionally, probabilistic ICA applied to neuro-oncology allowed the detection of abnormal tumor vasculature, distinguishing tumor from normal tissue and potentially serving as a biomarker to predict responses to antiangiogenic drugs. ${ }^{22,40}$ The present study suggests that the use of this method can be expanded to surgical planning and monitoring for EDAS in patients with ICAS. Despite recent concern about gadolinium deposits after repeat use, postcontrast MR imaging is standard in the stroke protocols of many institutions and DSC-MR imaging can be performed concurrently without additional contrast administration. ${ }^{41,42}$ While the clinical significance of gadolinium deposits after repeat use still remains unknown, the application of probabilistic ICA to DSC-MR imaging may prove beneficial for surgical planning and monitoring for EDAS. ${ }^{41,42}$ These potential benefits may thus outweigh the risk of gadolinium; however, further investigation is warranted.

\section{CONCLUSIONS}

The current results indicate that probabilistic ICA of DSC-MR imaging data is a sensitive technique for monitoring changes in perfusion after EDAS surgery in patients with ICAS and MMD. Hypoperfused brain regions had a high probability of perfusion characteristics, consistent with venous hemodynamics, and this elevated probability decreased as newly formed collaterals became evident.

Disclosures: Nestor R. Gonzalez-RELATED: Grant: National Institutes of Health, Comments: National Institutes of Health, National Institute of Neurological Disorders and Stroke, K23NS079477-01A1. * *Money paid to the institution.

\section{REFERENCES}

1. Adelson PD, Scott RM. Pial synangiosis for Moyamoya syndrome in children. Pediatr Neurosurg 1995;23;26-33 Medline

2. Matsushima $\mathrm{T}$, Inoue $\mathrm{T}$, Ikezaki $\mathrm{K}$, et al. Multiple combined indirect procedure for the surgical treatment of children with Moyamoya disease: a comparison with single indirect anastomosis and direct anastomosis. Neurosurg Focus 1998;5; 4 Medline

3. Sainte-Rose C, Oliveira R, Puget S, et al. Multiple bur hole surgery for the treatment of Moyamoya disease in children. J Neurosurg 2006;105(6 suppl):437-43 Medline

4. Scott RM, Smith JL, Robertson RL, et al. Long-term outcome in children with moyamoya syndrome after cranial revascularization by pial synangiosis. J Neurosurg 2004;100(2 suppl pediatrics):142-49 Medline

5. Dusick JR, Gonzalez NR, Martin NA. Clinical and angiographic outcomes from indirect revascularization surgery for Moyamoya disease in adults and children: a review of 63 procedures. Neurosurgery 2011;68:34-43; discussion 43 CrossRef Medline

6. Dusick JR, Liebeskind DS, Saver JL, et al. Indirect revascularization for nonmoyamoya intracranial arterial stenoses: clinical and angiographic outcomes. J Neurosurg 2012;117:94-102 CrossRef Medline

7. Gonzalez NR, Liebeskind DS, Dusick JR, et al. Intracranial arterial stenoses: current viewpoints, novel approaches, and surgical perspectives. Neurosurg Rev 2013;36:175-84; discussion 184-85 CrossRef Medline

8. Starke RM, Komotar RJ, Connolly ES. Optimal surgical treatment for Moyamoya disease in adults: direct versus indirect bypass. $\mathrm{Neu}$ rosurg Focus 2009;26:E8 CrossRef Medline

9. Gonzalez NR, Dusick JR, Connolly M, et al. Encephaloduroarteriosynangiosis for adult intracranial arterial steno-occlusive disease: long-term single-center experience with 107 operations. J Neurosurg 2015;123:654-61 CrossRef Medline

10. Chimowitz MI, Lynn MJ, Derdeyn CP, et al; SAMMPRIS Trial Investigators. Stenting versus aggressive medical therapy for intracranial arterial stenosis. N Engl J Med 2011;365:993-1003 CrossRef Medline

11. Derdeyn CP, Chimowitz MI, Lynn MJ, et al; Stenting and Aggressive Medical Management for Preventing Recurrent Stroke in Intracranial Stenosis Trial Investigators. Aggressive medical treatment with or without stenting in high-risk patients with intracranial artery stenosis (SAMMPRIS): the final results of a randomised trial. Lancet 2014;383:333-41 CrossRef Medline

12. Ginat DT, Smith ER, Robertson RL, et al. Imaging after direct and indirect extracranial-intracranial bypass surgery. $A J R$ Am J Roentgenol 2013;201:W124-32 CrossRef Medline

13. Blauwblomme T, Lemaitre H, Naggara O, et al. Cerebral blood flow improvement after indirect revascularization for pediatric Moyamoya disease: a statistical analysis of arterial spin-labeling MRI. AJNR Am J Neuroradiol 2016;37:706-12 CrossRef Medline

14. Yoon HK, Shin HJ, Lee M, et al. MR angiography of moyamoya disease before and after encephaloduroarteriosynangiosis. AJR Am J Roentgenol 2000;174:195-200 CrossRef Medline

15. Robertson RL, Burrows PE, Barnes PD, et al. Angiographic changes AJNR Am J Neuroradiol 38:507-14 Mar 2017 www.ajnr.org 513 
after pial synangiosis in childhood moyamoya disease. AJNR Am J Neuroradiol 1997;18:837-45 Medline

16. Lee SK, Kim DI, Jeong EK, et al. Postoperative evaluation of moyamoya disease with perfusion-weighted MR imaging: initial experience. AJNR Am J Neuroradiol 2003;24:741-47 Medline

17. Liebeskind DS, Sanossian N. How well do blood flow imaging and collaterals on angiography predict brain at risk? Neurology 2012;79: S105-09 CrossRef Medline

18. Sam K, Poublanc J, Sobczyk O, et al. Assessing the effect of unilateral cerebral revascularization on the vascular reactivity of the non-intervened hemisphere: a retrospective observational study. $B M J$ Open 2015;5:e006014 CrossRef Medline

19. Oh SW, Kim YK, Kim SK, et al. Hemodynamic improvement of anterior cerebral artery territory perfusion induced by bifrontal encephalo(periosteal) synangiosis in pediatric patients with moyamoya disease: a study with brain perfusion SPECT. Ann Nucl Med 2012;26:47-57 CrossRef Medline

20. Dai DW, Zhao WY, Zhang YW, et al. Role of CT perfusion imaging in evaluating the effects of multiple burr hole surgery on adult ischemic Moyamoya disease. Neuroradiology 2013;55:1431-38 CrossRef Medline

21. Lu CF, Chou YC, Guo WY, et al. Brain MR perfusion image segmentation using independent component analysis and hierarchical clustering. Conf Proc IEEE Eng Med Biol Soc 2007;2007:5547-50 Medline

22. LaViolette PS, Cohen AD, Prah MA, et al. Vascular change measured with independent component analysis of dynamic susceptibility contrast MRI predicts bevacizumab response in high-grade glioma. Neuro Oncol 2013;15:442-50 CrossRef Medline

23. Damoiseaux JS, Rombouts SA, Barkhof F, et al. Consistent restingstate networks across healthy subjects. Proc Natl Acad Sci U S A 2006;103:13848-53 CrossRef Medline

24. Calhoun VD, Liu J, Adali T. A review of group ICA for fMRI data and ICA for joint inference of imaging, genetic, and ERP data. Neuroimage 2009;45:S163-72 CrossRef Medline

25. Onton J, Westerfield M, Townsend J, et al. Imaging human EEG dynamics using independent component analysis. Neurosci Biobehav Rev 2006;30:808-22 CrossRef Medline

26. Delorme A, Makeig S. EEGLAB: an open source toolbox for analysis of single-trial EEG dynamics including independent component analysis. J Neurosci Methods 2004;134:9-21 CrossRef Medline

27. Smith SM, Fox PT, Miller KL, et al. Correspondence of the brain's functional architecture during activation and rest. Proc Natl Acad Sci U S A 2009;106:13040 - 45 CrossRef Medline

28. Beckmann CF, Smith SM. Probabilistic independent component analysis for functional magnetic resonance imaging. IEEE Trans Med Imaging 2004;23:137-52 CrossRef Medline

29. Nichols K, Holmes A. Non-parametric procedures. Statistical Parametric Mapping 2007;253-72 CrossRef
30. Macchi C, Catini C, Federico C, et al. Magnetic resonance angiographic evaluation of circulus arteriosus cerebri (circle of Willis): a morphologic study in $\mathbf{1 0 0}$ human healthy subjects. Ital J Anat Embryol 1996;101:115-23 Medline

31. Lee SC, Jeon JS, Kim JE, et al. Contralateral progression and its risk factor in surgically treated unilateral adult moyamoya disease with a review of pertinent literature. Acta Neurochir (Wien) 2014;156: 103-11 CrossRef Medline

32. Amin-Hanjani S, Singh A, Rifai H, et al. Combined direct and indirect bypass for moyamoya: quantitative assessment of direct bypass flow over time. Neurosurgery 2013;73: 962-67; discussion 967-68 CrossRef Medline

33. Ryu WS, Park SS, Kim YS, et al. Long-term natural history of intracranial arterial stenosis: an MRA follow-up study. Cerebrovasc Dis 2014;38:290-96 CrossRef Medline

34. Kim YI, Phi JH, Paeng JC, et al. In vivo evaluation of angiogenic activity and its correlation with efficacy of indirect revascularization surgery in pediatric Moyamoya disease. J Nucl Med 2014;55: 1467-72 CrossRef Medline

35. Calamante F, Gadian DG, Connelly A. Delay and dispersion effects in dynamic susceptibility contrast MRI: simulations using singular value decomposition. Magn Reson Med 2000;44:466-73 Medline

36. Wu O, Østergaard L, Weisskoff RM, et al. Tracer arrival timinginsensitive technique for estimating flow in MR perfusionweighted imaging using singular value decomposition with a blockcirculant deconvolution matrix. Magn Reson Med 2003;50:164-74 CrossRef Medline

37. Kao YH, Teng MM, Liu KC, et al. Hemodynamic segmentation of MR perfusion images in patients with unilateral carotid stenosis using independent component analysis. J Magn Reson Imaging 2008; 28:1125-32 CrossRef Medline

38. McKeown MJ, Makeig S, Brown GG, et al. Analysis of fMRI data by blind separation into independent spatial components. Hum. Brain Mapp 1998;6:160-88 Medline

39. Kao YH, Guo WY, Wu YT, et al. Hemodynamic segmentation of MR brain perfusion images using independent component analysis, thresholding, and Bayesian estimation. Magn Reson Med 2003;49: 885-94 CrossRef Medline

40. LaViolette PS, Daun MK, Paulson ES, et al. Effect of contrast leakage on the detection of abnormal brain tumor vasculature in highgrade glioma. J Neurooncol 2014;16:543-49 CrossRef Medline

41. Ramalho J, Semelka RC, Ramalho M, et al. Gadolinium-based contrast agent accumulation and toxicity: an update. AJNR Am J Neuroradiol 2016;37:1192-98 CrossRef Medline

42. Stojanov D, Aracki-Trenkic A, Benedeto-Stojanov D. Gadolinium deposition within the dentate nucleus and globus pallidus after repeated administrations of gadolinium-based contrast agents-current status. Neuroradiology 2016;58:433-441 CrossRef Medline 